\title{
NMR and IR study of fluorobenzene and hexafluorobenzene adsorbed on
}

\author{
alumina
}

Vitaliy L. Budarin, James H. Clark, Sarah E. Hale, Stewart J. Tavener* and Karl T. Mueller, Nancy M. Washton

\section{Supporting information S1.}

Nitrogen adsorption data and pore size distribution for neutral alumina. 
Adsorbate Property Factor: 9.530000

Fraction of Pores Open at Both Ends: 0.000

\begin{tabular}{|c|c|c|c|c|c|}
\hline $\begin{array}{c}\text { Average } \\
\text { Diameter } \\
\text { (A) }\end{array}$ & $\begin{array}{c}\text { Cumulative } \\
\text { Pore Volume } \\
\left.\text { (cm } \mathrm{Cm}^{3} / \mathrm{g}\right)\end{array}$ & $\begin{array}{l}\text { Smoothed } \\
\text { dV/dD } \\
\text { Pore Volume } \\
\text { (cin } / / \mathrm{g}-\mathrm{A})\end{array}$ & $\begin{array}{l}\text { Cumulative } \\
\text { Pore Area } \\
\left(\mathbb{I}^{2} / g\right)\end{array}$ & $\begin{array}{l}\text { Smoothed } \\
\text { dA } / \mathrm{dD} \\
\text { Pore Area } \\
\left(\mathrm{IL}^{2} / \mathrm{g}-\mathrm{A}\right)\end{array}$ & $\begin{array}{l}\text { Smoothed } \\
\text { dV/dlog (D) } \\
\text { Pore Volume } \\
\left.\text { (cm } \text { (cm }^{3} / \mathrm{g}^{-1}\right)\end{array}$ \\
\hline 2378.6 & 0.006943 & $5.7042 e-06$ & 0.117 & $1.1213 \mathrm{e}-04$ & $2.6216 \mathrm{e}-02$ \\
\hline 1276.6 & 0.015520 & $1.4799 \mathrm{e}-05$ & 0.385 & $5.4520 \mathrm{e}-04$ & $3.6571 \mathrm{e}-02$ \\
\hline 862.3 & 0.021782 & $2.6647 e-05$ & 0.676 & $\begin{array}{l}1.4972 \mathrm{e}-03 \\
\end{array}$ & $4.6550 \mathrm{e}-02$ \\
\hline 471.0 & 0.036355 & $6.2473 e-05$ & 1.913 & $6.5990 \mathrm{e}-03$ & $5.6952 \mathrm{e}-02$ \\
\hline 306.7 & 0.046425 & $9.9301 \mathrm{e}-05$ & 3.227 & 1. $5487 \mathrm{e}-02$ & $6.1124 \mathrm{e}-02$ \\
\hline 224.5 & 0.054068 & $1.3273 e-04$ & 4.589 & $2.7202 \mathrm{e}-02$ & $6.1435 e-02$ \\
\hline 176.9 & 0.059831 & $1.5976 \mathrm{e}-04$ & 5.892 & $4.0242 \mathrm{e}-02$ & $5.9479 \mathrm{e}-02$ \\
\hline 145.2 & 0.064572 & $1.9124 \mathrm{e}-04$ & 7.198 & $5.7655 \mathrm{e}-02$ & $5.9040 \mathrm{e}-02$ \\
\hline 127.4 & 0.067036 & 2. $1353 \mathrm{e}-04$ & 7.972 & 7. $1348 \mathrm{e}-02$ & $5.9960 \mathrm{e}-02$ \\
\hline 108.0 & 0.072498 & 2. $9989 \mathrm{e}-04$ & 9.996 & $1.2455 \mathrm{e}-01$ & $6.8378 \mathrm{e}-02$ \\
\hline 88.3 & 0.079506 & $6.1991 \mathrm{e}-04$ & 13.170 & $3.2225 \mathrm{e}-01$ & $1.1600 \mathrm{e}-01$ \\
\hline 72.0 & 0.096422 & $1.9599 \mathrm{e}-03$ & 22.565 & $1.1781 \mathrm{e}+00$ & $2.9853 \mathrm{e}-01$ \\
\hline 59.6 & 0.126174 & $3.2823 \mathrm{e}-03$ & 42.529 & $2.4617 \mathrm{e}+00$ & $4.1734 \mathrm{e}-01$ \\
\hline 50.6 & 0.155502 & 3. $7268 \mathrm{e}-03$ & 65.718 & $3.1483 \mathrm{e}+00$ & 4. $0605 \mathrm{e}-01$ \\
\hline 43.7 & 0.178159 & $3.4726 \mathrm{e}-03$ & 86.440 & $3.3919 \mathrm{e}+00$ & $3.2876 \mathrm{e}-01$ \\
\hline 38.2 & 0.194599 & $2.9933 \mathrm{e}-03$ & 103.656 & $3.3255 \mathrm{e}+00$ & 2. $4825 \mathrm{e}-01$ \\
\hline 33.4 & 0.206810 & 2. $4868 \mathrm{e}-03$ & 118.260 & $3.1487 \mathrm{e}+00$ & $1.8051 \mathrm{e}-01$ \\
\hline 29.7 & 0.214315 & $2.1043 \mathrm{e}-03$ & 128.383 & $2.9652 \mathrm{e}+00$ & 1. $3660 \mathrm{e}-01$ \\
\hline 27.8 & 0.215701 & $2.0342 \mathrm{e}-03$ & 130.375 & $2.9316 \mathrm{e}+00$ & 1. $2889 \mathrm{e}-01$ \\
\hline 27.2 & 0.217040 & $1.9659 \mathrm{e}-03$ & 132.346 & $2.9009 \mathrm{e}+00$ & $1.2148 \mathrm{e}-01$ \\
\hline 26.5 & 0.218223 & $1.9038 \mathrm{e}-03$ & $\begin{array}{l}134.131 \\
\end{array}$ & $2.8742 \mathrm{e}+00$ & $1.1491 \mathrm{e}-01$ \\
\hline 25.9 & 0.219353 & $1.8449 \mathrm{e}-03$ & 135.875 & $2.8493 \mathrm{e}+00$ & $1.0886 \mathrm{e}-01$ \\
\hline 25.3 & 0.220304 & $1.7832 \mathrm{e}-03$ & 137.378 & $2.8235 \mathrm{e}+00$ & $1.0272 \mathrm{e}-01$ \\
\hline 24.7 & 0.221359 & $1.7255 \mathrm{e}-03$ & 139.085 & $2.7997 \mathrm{e}+00$ & $9.7027 \mathrm{e}-02$ \\
\hline 24.1 & 0.222349 & $1.6702 \mathrm{e}-03$ & 140.727 & $2.7771 \mathrm{e}+00$ & $9.1668 \mathrm{e}-02$ \\
\hline 23.5 & 0.223281 & $1.6189 \mathrm{e}-03$ & 142.310 & $2.7585 \mathrm{e}+00$ & $8.6707 \mathrm{e}-02$ \\
\hline 23.0 & 0.224208 & 1. $5710 \mathrm{e}-03$ & 143.924 & $2.7439 \mathrm{e}+00$ & $8.2048 \mathrm{e}-02$ \\
\hline 22.4 & 0.225032 & $1.5253 \mathrm{e}-03$ & 145.396 & $2.7295 \mathrm{e}+00$ & 7. $7772 \mathrm{e}-02$ \\
\hline 21.9 & 0.225823 & $1.4809 \mathrm{e}-03$ & $\begin{array}{l}146.843 \\
146.843\end{array}$ & $2.7134 \mathrm{e}+00$ & $7.3703 e-02$ \\
\hline 21.3 & 0.226568 & 1. $4317 \mathrm{e}-03$ & 148.239 & $2.6883 \mathrm{e}+00$ & $6.9529 \mathrm{e}-02$ \\
\hline 20.8 & 0.227334 & 1. $3801 \mathrm{e}-03$ & 149.711 & $2.6590 \mathrm{e}+00$ & $6.5240 \mathrm{e}-02$ \\
\hline 20.3 & 0.228014 & 1. $3308 \mathrm{e}-03$ & 151.053 & $2.6303 \mathrm{e}+00$ & $6.1349 \mathrm{e}-02$ \\
\hline 19.8 & 0.228671 & $1.2860 \mathrm{e}-03$ & 152.383 & $2.6056 \mathrm{e}+00$ & $5.7875 \mathrm{e}-02$ \\
\hline 19.3 & 0.229275 & $1.2449 \mathrm{e}-03$ & 153.635 & $2.5899 \mathrm{e}+00$ & $5.4569 \mathrm{e}-02$ \\
\hline 18.8 & 0.229862 & $1.2063 e-03$ & 154.885 & $2.5789 \mathrm{e}+00$ & $5.1523 e-02$ \\
\hline $\begin{array}{l}10.0 \\
18.3\end{array}$ & 0.230430 & $1.1673 e-03$ & $\begin{array}{l}156.126 \\
1560\end{array}$ & $2.5652 \mathrm{e}+00$ & $4.8523 e-02$ \\
\hline 17.8 & 0.230962 & $1.1254 \mathrm{e}-03$ & 157.324 & 2. $5436 \mathrm{e}+00$ & 4. $5493 \mathrm{e}-02$ \\
\hline 17.3 & 0.231457 & $1.0815 \mathrm{e}-03$ & 158.466 & $2.5073 \mathrm{e}+00$ & $4.2576 \mathrm{e}-02$ \\
\hline 16.6 & 0.232529 & $9.8452 \mathrm{e}-04$ & 161.052 & 2. $4128 \mathrm{e}+00$ & $3.6555 \mathrm{e}-02$ \\
\hline 15.6 & 0.233374 & $8.8505 \mathrm{e}-04$ & 163.213 & $2.2947 \mathrm{e}+00$ & $3.1015 \mathrm{e}-02$ \\
\hline 14.7 & 0.234086 & $7.7225 \mathrm{e}-04$ & 165.149 & $2.1389 \mathrm{e}+00$ & $2.5388 \mathrm{e}-02$ \\
\hline 13.8 & 0.234749 & $6.7691 \mathrm{e}-04$ & $\begin{array}{l}167.075 \\
167.075\end{array}$ & $2.0173 \mathrm{e}+00$ & $2.0777 \mathrm{e}-02$ \\
\hline 12.8 & 0.2353 & $5.9957 \mathrm{e}-04$ & 168.992 & $1.9213 \mathrm{e}+00$ & 1. $7076 \mathrm{e}-02$ \\
\hline 11.8 & 0.235953 & $5.1143 \mathrm{e}-$ & 170.991 & 1. $7996 \mathrm{e}+00$ & 1. $3307 \mathrm{e}-02$ \\
\hline 10.7 & 0.236437 & $4.6107 \mathrm{e}-04$ & 172.801 & 1. $8100 \mathrm{e}+00$ & 1. $0832 \mathrm{e}-02$ \\
\hline 9.7 & 0.236886 & $4.9800 \mathrm{e}-04$ & 174.664 & $2.1780 \mathrm{e}+00$ & $1.0569 \mathrm{e}-02$ \\
\hline 8.6 & 0.237489 & $6.7417 \mathrm{e}$ & 177.471 & $3.4099 \mathrm{e}+00$ & $1.2589 \mathrm{e}-02$ \\
\hline 7.0 & & & & $\begin{array}{l}1.08 \\
1.08\end{array}$ & 2.47 \\
\hline 6.1 & & & & 1.4 & 2.90 \\
\hline 5. & & & & & \\
\hline 5. & & & & & \\
\hline 5.3 & & & & & 3.7971e-02 \\
\hline 5.0 & 0.243339 & 3.66 & 217.361 & $3.0371 \mathrm{e}+01$ & $4.0673 \mathrm{e}-02$ \\
\hline 4.4 & 0.246859 & $5.1590 \mathrm{e}-03$ & 249.662 & $5.3167 \mathrm{e}+01$ & $4.7725 \mathrm{e}-02$ \\
\hline 3.6 & 0.250979 & $6.2517 \mathrm{e}-03$ & 295.146 & $7.4449 \mathrm{e}+01$ & $4.7855 \mathrm{e}-02$ \\
\hline 3.0 & 0.254761 & $5.3123 \mathrm{e}-03$ & 345.928 & $7.7634 \mathrm{e}+01$ & $3.3210 \mathrm{e}-02$ \\
\hline 2.4 & 0.256633 & 1. $6755 \mathrm{e}-03$ & 377.655 & $3.6702 \mathrm{e}+01$ & $8.0885 \mathrm{e}-03$ \\
\hline
\end{tabular}




\section{BJH Adsorption Cumulative Pore Volume}

+ Cumulative Pore Volume

$\circ \mathrm{dV} / \mathrm{dD}$ Pore Volume

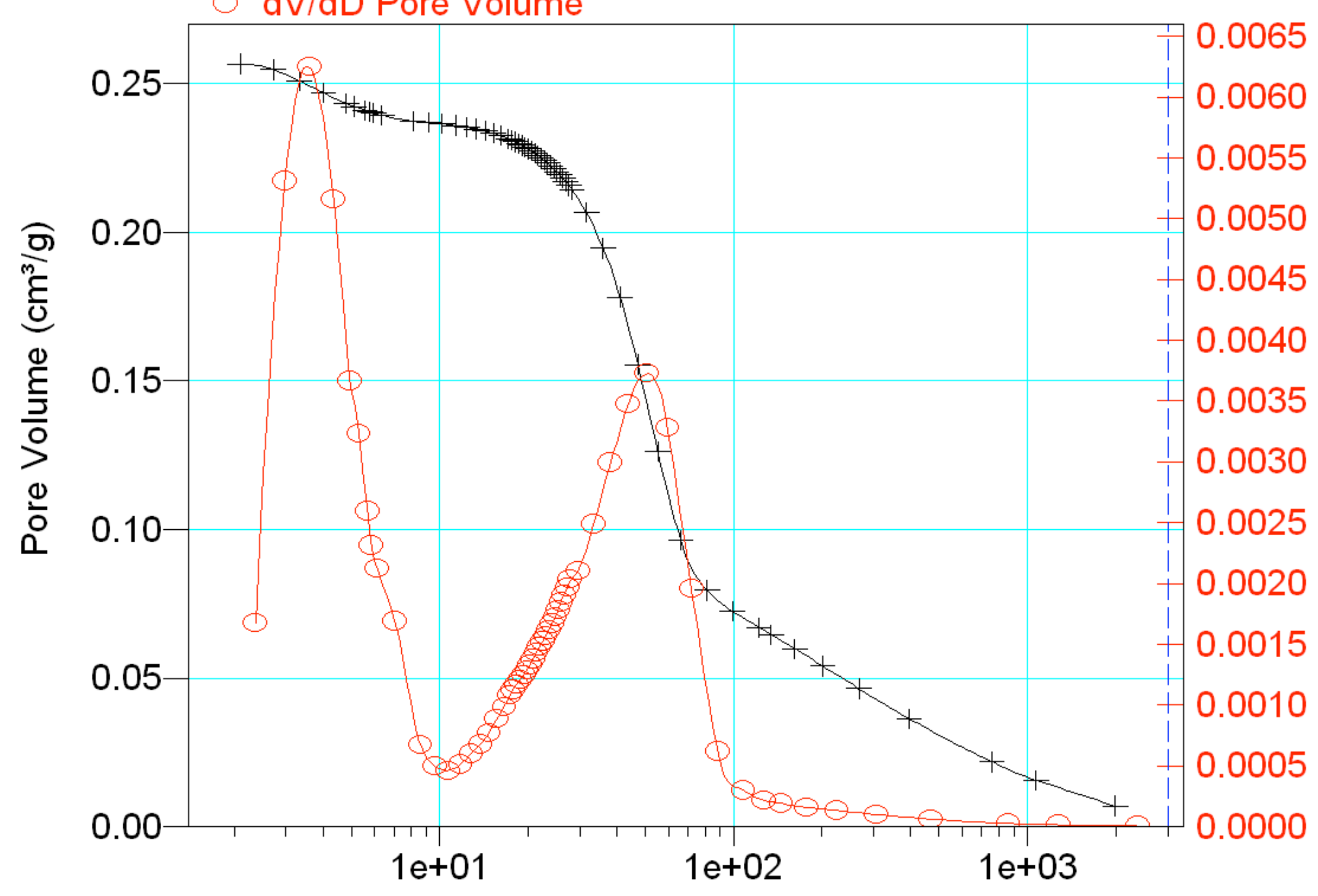

Pore Diameter $(A)$ 


\section{BJH Adsorption dV/dlog(D) Pore Volume} (smoothed)

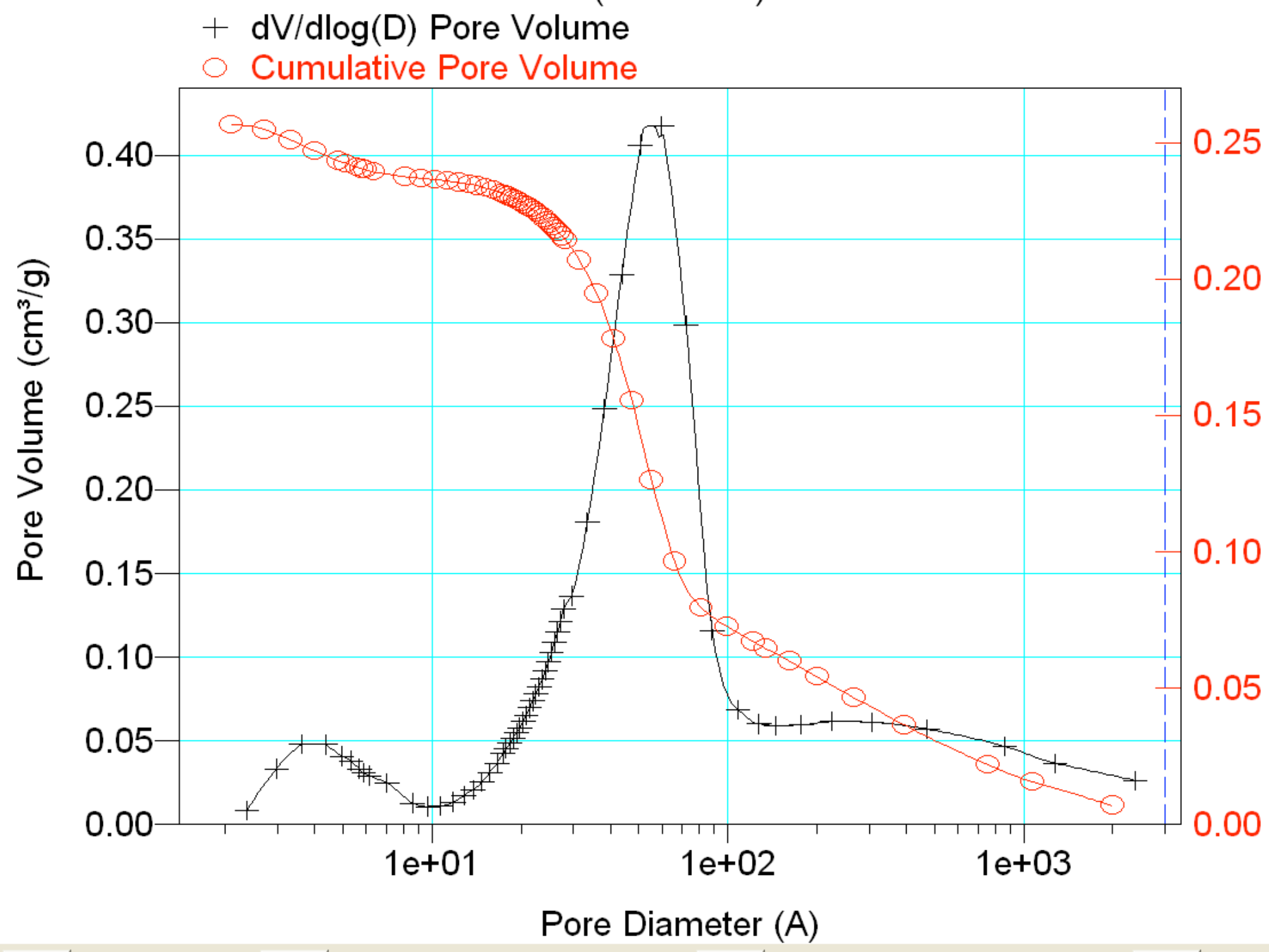

